\title{
Transferencia de conocimiento e innovación social en el desarrollo de huertos comunitarios mediados por tecnologías
}

\author{
Giraldo-Pinedo, Diana Marcela, Mg. ${ }^{1}$, Núñez-Velasco, Juan Manuel, $\mathrm{Mg}^{2}$, and Paredes-Chain, Ana Judith, Phd ${ }^{3}$ \\ ${ }^{1}$ Giraldo-Pinedo, Diana Marcela, Paredes-Chacin, Ana Judith, Universidad Autónoma de Occidente, Colombia \\ dmgiraldo@uao.edu.co,ajparedes@uao.edu.co \\ ${ }^{2}$ Núñez-Velasco, Juan Manuel, Universidad Autónoma de Occidente, Colombia,jmnunez@uao.edu.co
}

\begin{abstract}
Resumen-La relevancia de la transferencia de conocimiento se afianza en la pertinencia de su producción y utilidad, por lo cual el objetivo del estudio es determinar como la transferencia de conocimiento e innovación social soportan el desarrollo de huertos comunitarios mediados por tecnologías, como estrategia que contribuye con la soberanía alimentaria en comunidades vulneradas. El método responde a una investigación de campo, estructurada en tres fases: primera el análisis del contexto e identificación de capacidades y recursos, la segunda asociada con las transferencia de conocimiento científico-técnico para co-crear y la tercera basada en la aplicación de metodologías de diseño electrónico y de innovación basada en la industria 4.0. La aplicación del diseño se consolida de forma conjunta entre una institución de educación superior $y$ un sector comunitario del distrito de Aguablanca con una población de 16.353 habitantes en Santiago de Cali-Colombia. Los resultados determinan la existencia de huertos comunitarios tradicionales, sin considerar procesos de transferencia de conocimiento que debilitan la creación de huertos sostenibles mediados por tecnologías para fortalecer la capacidad de producción de hortalizas y frutales. El prototipo fue diseñado bajo criterios sostenibles, lo cual permite su apropiación garantizando la autogestión por las comunidades. Se concluye sobre una innovación social factible que transforma contextos para la producción de alimentos minimizando impactos socio ambientales.
\end{abstract}

Palabras clave - transferencia de conocimiento; innovación social; habilidades técnicas; huertos sostenibles; soberanía alimentaria.

\footnotetext{
Abstract-The relevance of knowledge transfer is based on the pertinence of its production and usefulness, so the objective of the study is to determine how knowledge transfer and social innovation support the development of technology-mediated community gardens as a strategy that contributes to food sovereignty in vulnerable communities. The method responds to a field research, structured in three phases: first the analysis of the context and identification of capacities and resources, the second associated with the transfer of scientific-technical knowledge to co-create and the third based on the application of methodologies of electronic design and innovation based on industry 4.0. The application of the model is consolidated jointly with a university and commune 13 of the Aguablanca district with a population of 16,353 inhabitants in Cali-Colombia. The results determine the existence of traditional community gardens with weak knowledge transfer processes that weaken the creation of sustainable gardens mediated by technologies to strengthen the production capacity of vegetables and fruit trees. The prototype designed responds to an accessible model. The appropriation of the
}

model guarantees self-management by the communities, concluding on a feasible social innovation that transforms contexts for food production while minimizing socio-environmental impacts.

Keywords - knowledge transfer; social innovation; technical skills; sustainable gardens; food sovereignty.
Digital Object Identifier (DOI):

http://dx.doi.org/10.18687/LEIRD2021.1.1.14

ISSN: 2414-6390 ISBN: 978-958-52071-9-6 


\title{
Transferencia de conocimiento e innovación social en el desarrollo de huertos comunitarios mediados por tecnologías
}

\author{
Giraldo-Pinedo, Diana Marcela, Mg. ${ }^{1}$, Núñez-Velasco, Juan Manuel, $\mathrm{Mg}^{2}$, and Paredes-Chain, Ana Judith, Phd ${ }^{3}$ \\ ${ }^{1}$ Giraldo-Pinedo, Diana Marcela, Paredes-Chacin, Ana Judith, Universidad Autónoma de Occidente, Colombia \\ dmgiraldo@uao.edu.co,ajparedes@uao.edu.co \\ ${ }^{2}$ Núnez-Velasco, Juan Manuel, Universidad Autónoma de Occidente, Colombia, jmnunez@uao.edu.co
}

\begin{abstract}
Resumen- La relevancia de la transferencia de conocimiento se afianza en la pertinencia de su producción y utilidad, por lo cual el objetivo del estudio es determinar como la transferencia de conocimiento e innovación social soportan el desarrollo de huertos comunitarios mediados por tecnologías, como estrategia que contribuye con la soberanía alimentaria en comunidades vulneradas. El método responde a una investigación de campo, estructurada en tres fases: primera el análisis del contexto e identificación de capacidades y recursos, la segunda asociada con las transferencia de conocimiento científico-técnico para co-crear y la tercera basada en la aplicación de metodologías de diseño electrónico y de innovación basada en la industria 4.0. La aplicación del diseño se consolida de forma conjunta entre una institución de educación superior y un sector comunitario del distrito de Aguablanca con una población de 16.353 habitantes en Santiago de Cali-Colombia. Los resultados determinan la existencia de huertos comunitarios tradicionales, sin considerar procesos de transferencia de conocimiento que debilitan la creación de huertos sostenibles mediados por tecnologías para fortalecer la capacidad de producción de hortalizas y frutales. El prototipo fue diseñado bajo criterios sostenibles, lo cual permite su apropiación garantizando la autogestión por las comunidades. Se concluye sobre una innovación social factible que transforma contextos para la producción de alimentos minimizando impactos socio ambientales.
\end{abstract}

Palabras clave - transferencia de conocimiento; innovación social; habilidades técnicas; huertos sostenibles; soberanía alimentaria.

Abstract-The relevance of knowledge transfer is based on the pertinence of its production and usefulness, so the objective of the study is to determine how knowledge transfer and social innovation support the development of technology-mediated community gardens as a strategy that contributes to food sovereignty in vulnerable communities. The method responds to a field research, structured in three phases: first the analysis of the context and identification of capacities and resources, the second associated with the transfer of scientific-technical knowledge to co-create and the third based on the application of methodologies of electronic design and innovation based on industry 4.0. The application of the model is consolidated jointly with a university and commune 13 of the Aguablanca district with a population of 16,353 inhabitants in Cali-Colombia. The results determine the existence of traditional community gardens with weak knowledge transfer processes that weaken the creation of sustainable gardens mediated by technologies to strengthen the production capacity of vegetables and fruit trees. The prototype designed responds to an accessible model. The appropriation of the $1^{\text {sh }}$ LACCEI International Multiconference on Entrepreneurship, Innovation and Regional Development - LEIRD 2021: "Ideas to Overcome and Emerge from the Pandemic Crisis", Virtual Edition, December 9 - 10, 2021. model guarantees self-management by the communities, concluding on a feasible social innovation that transforms contexts for food production while minimizing socio-environmental impacts.

Keywords- knowledge transfer; social innovation; technical skills; sustainable gardens; food sovereignty.

\section{INTRODUCCIÓN}

Las transformaciones que surgen en determinados entornos sociales, frecuentemente vulnerados por debilidades de producción y dominio de conocimientos, han sido considerados como variables que ameritan ser analizadas con el fin de promover mediante interacciones entre universidad-sociedad, acciones que contribuyan con la generación de valor y proyecten alternativas que impulsen la autogestión en entornos sociales. De esta forma, se generan alternativas que fomentan iniciativas centradas en la innovación social, con objetivos sociales direccionadas a transformar la realidad antes expuesta [1]. La importancia que fundamenta el desarrollo del estudio, se centra en las formas de garantizar, no solo la producción de conocimiento, en este caso generado desde las instituciones de educación superior, en adelante IES, sino también las formas de hacer efectiva su difusión y la transferencia en el marco del cumplimiento de la misión de las IES [2] para promover la innovación social.

Lo descrito, reafirma la necesidad de trascender de las aulas académicas para promover la transferencia de conocimiento científico-técnico e impulsar la apropiación del conocimiento por parte de las comunidades, previa interacción entre investigadores - sociedad que logran apropiarse del conocimiento científico-tecnológico mediante prácticas. Bajo esta perspectiva, se representa la sistematización de una experiencia, consolidada a partir de la transferencia de conocimiento y la co-creación como base para fomentar la innovación social, representada en el desarrollo de huertos comunitarios mediados por tecnologías. Para su estructura, se definen características accesibles del diseño en el marco de la sostenibilidad, lo cual le otorga la viabilidad de un proyecto de innovación social que permite transformar y garantizar en cierta medida la seguridad alimentaria de la comunidad. 
La efectividad de procesos de interacción entre universidad y sociedad, permite desarrollar el objetivo de determinar como la transferencia de conocimiento e innovación social soportan el desarrollo de huertos comunitarios mediados por tecnologías, como estrategia que contribuye con la soberanía alimentaria en comunidades vulneradas. $\mathrm{Su}$ práctica evidencia las interacciones con la comunidad y la capacidad de esta de apropiación del conocimiento, a partir de conceptos asociados con los requerimientos de diseño, desarrollo de objetos acorde al contexto, construcción y manejo de tecnología para el diseño de prototipos basados en agricultura de precisión. Al respecto, lo más importante es el monitoreo y trazabilidad de variables agroclimáticas, entre las cuales su orden de prioridad se basan en un estudio previo dependiendo del tipo de cultivo, el tipo de suelo, como también la medición de variables durante el semillero y cosecha. Para la estructura de contenido del estudio se presenta en una primera fase la revisión de la literatura asociadas con la transferencia de conocimiento, innovación social y el desarrollo de huertos, en segunda fase, se describe el método, considerando las fases de desarrollo y en tercera fase se presenta el análisis de resultados fundamentado en el diseño del modelo propuesto y ejecutado para dar continuidad con las conclusiones, implicaciones prácticas y líneas de investigación futuras.

\section{CONTEXTO}

\section{A. Transferencia de conocimiento como estrategia de interacción universidad-sociedad}

La transferencia de conocimiento a partir de su efectiva producción se ha convertido en la última década en un recurso estratégico mediante el cual, se impulsan investigaciones, nuevos desarrollos, innovaciones y emprendimientos. Entre las teorías fundamentadas sobre la gestión del conocimiento, está implícito las formas de producción y de transferencia basada en la capacidad de crear, procesar, transmitir y por ende lograr la absorción y explotación de este recurso [3][4][5][6].

Como aspecto determinante se resalta la trilogía del proceso producción-transferencia-apropiación social del conocimiento [7]. Proceso que se consolida, mediante las interacciones sociales en las que prevalecieron procesos de comunicación efectivos entre diferentes actores y a partir de capacidades y recursos disponibles se promueven actividades formativas que sobre el presente estudio dieron paso a un desarrollo articulado que conlleva a la innovación social. Esta se concibe entre otros enfoques por la responsabilidad de garantizar la solidaridad y la igualdad de condiciones de vida para los más vulnerables [8]. De esta forma, la viabilidad de la innovación social propuesta estuvo enfocada en una visión de intereses compartidos entre la academia y la sociedad. El enfoque sobre su alcance, desde el fundamento teórico de tres componentes de la quíntuple hélice [9] [10] centró el énfasis de relaciones entre la universidad: como institución que lidera procesos de producción y transferencia de conocimiento, la sociedad: en la que intervienen los actores de las comunidades e interactúan, logrando la apropiación social del conocimiento para transformarlo en beneficios colectivos, y el ambiente: visto ante la inaplazable búsqueda de alternativas que proyecten minimizar los impactos que se generan y afectan todo el ecosistema ambiental. A partir de lo expuesto, el enfoque que consolida la quíntuple hélice mediante la cual se extiende la Hélice Cuádruple por aspectos de los "entornos naturales de la sociedad y la economía", la "ecología social" y la "transición socioecológica" [11].

Desde la perspectiva del estudio, para el desarrollo y operatividad de huertos comunitarios, se prioriza: - el contexto de aplicación de la experiencia desde las instituciones en las que se produce el conocimiento científico-técnico, - las estrategias para garantizar la transferencia en entornos socio-comunitarios, - la visión de sostenibilidad que prevé la articulación de las dimensiones social, económica y ambiental que la determinan. Sumado a la necesidad de lograr la asociación de nuevas formas de viabilizar de interacción, no solo para el desarrollo de los huertos comunitarios, sino también para monitorear la efectividad funcional, la permanente renovación, considerando el seguimiento mediante sistemas y tecnologías que alerten sobre los riesgos e incertidumbres que surjan de los entornos.

Lo descrito responde a las características distintivas consideradas en el diseño del proyecto como una forma de garantizar desde la transferencia de conocimiento y su puesta en práctica beneficios asociados con la seguridad alimentaria, y en consecuencia contribuir con las demandas de un consumo responsable desde las comunidades. De esta forma, prevalece el compromiso con la estabilidad de la generación actual y futura. [12] [13]. Para tal efecto, los mecanismos de coordinación, hacen referencias al valor otorgado al conocimiento o las habilidades compartidas que orientan las acciones de los miembros de la comunidad quienes valoran y le otorgan valor a las interacciones entre la tecnología y la agricultura.

\section{B. El diseño social como garante de la apropiación de las soluciones por parte de la comunidad}

Tanto la innovación social como el diseño social invitan a la transformación de las estructuras las cuales conllevan a que un grupo de personas, no cuenten con soluciones de base resueltas, es por ello que invitan a que desde un ejercicio dinamizado por las comunidades exista una apropiación del conocimiento y de las dinámicas para el desarrollo de nuevas realidades en los territorios, la cual se da cuando el proceso surge desde y para la comunidad. El diseño para impulsar la innovación social es un proceso que debe ser colaborativo, donde el diseñador y "los expertos" utilicen sus conocimientos para reconocer los problemas en los cuales está sumergida la comunidad y que para que esa transformación de las estructuras que perpetúan la problemática se dé, las soluciones a las cuales se lleguen, deben 
ser más accesibles, eficaces, sostenibles y replicables que aquello con lo que hoy cuentan estos grupos poblacionales [14] [15].

Desde la perspectiva de diversos contextos de orden global y en el caso de colombiana, existen grandes grupos poblacionales con necesidad no resueltas, por lo que se hacen necesarias invitaciones de diseñadores sociales como [16] quienes no solo, invitan hacia el desarrollo de una nueva cultura que permita ver el mundo desde otra orilla, donde los ciudadanos sean activos en los procesos de transformación social, sino que también resaltan la relevancia de que esa transformación sea sociotécnica, lo cual permitiría la sostenibilidad de dicha evolución. Para tal efecto, se requiere que las comunidades adopten y se apropien de conocimientos para que las soluciones o nuevos caminos perduren en el tiempo y no se hagan dependientes de un actor externo para su continuidad.

\section{Pertinencia tecnológica en la agricultura urbana}

Actualmente las tecnologías aplicadas a la agricultura tradicional, huertos, jardines y agroindustria en Latinoamérica son en mayor parte artesanales (objetos existentes de los cuales se les da otro uso o técnicas ancestrales referente a prácticas agrícolas), lo cual no permite un desarrollo pleno de este sector. Debido a lo anterior, se hace necesario la aplicación de nuevas tecnologías en este campo con la finalidad de que puedan generar condiciones favorables de vida tanto para las personas involucradas en esta actividad. En muchas ocasiones los procesos de medición y seguimiento de los cultivos, independientemente si son huertos urbanos, rurales o cultivos de grandes extensiones, se realizan de forma manual y usando los sentidos como la vista y el tacto.

Entre las acciones que promueven incentivos para mediar la agricultura con tecnología, fue el hecho de procesos empíricos, mediante el cual se evidenció como un pequeño y mediano productor de arroz utiliza su dedo índice de la mano derecha para "sensar" si la tierra tenía mucha humedad o estaba bastante seca. Lo descrito, confirma una de las realidades y causales de por qué la agricultura en Latinoamérica se encuentra con muchas pérdidas en producción y sin poder mitigar los efectos del cambio climático de una manera eficiente. Ante lo descrito y su asociación con prácticas agrícolas débiles según el último censo[17] ratifica la importancia de pasar del machete a la computadora y se afirma que el $85 \%$ de los productores residentes en las áreas rurales dispersas, declararon no recibir asistencia técnica ni programas orientados a las huertas urbanas. Otro fenómeno que se presenta al no involucrar tecnologías mediadoras, es que se está envejeciendo la población rural y por ende falta de líderes para la creación de huertos urbanos. Entre otros datos del censo [18] la edad promedio de la población campesina oscila entre los 40 y 54 años. La preocupación del gobierno es evidente si se tiene en cuenta que de los 200.000 jóvenes bachilleres graduados en Colombia en el 2010, apenas
3.000 cursaron una carrera relacionada con el agro, el cual al pasar los años sigue bajando el índice de personas que quieren estudiar o realizar actividades agropecuarias, es decir no se está generando un relevo generacional para dichas actividades y mucho menos la migración del campo a la ciudad.

Lo antes descrito, requiere de procesos formativos que contribuyan con el desarrollo científico y tecnológico, sumado a la importancia sobre el seguimiento y la trazabilidad de las variables agroclimáticas en campo y en zonas urbanas, tales como la humedad relativa, humedad del suelo, temperatura ambiente, temperatura del suelo, luminosidad, radiación, $\mathrm{pH}$, velocidad del viento entre otras variables, tiene gran influencia en el rendimiento de los cultivos, en el crecimiento de la planta y todos los procesos bioquímicos y fisiológicos, como consecuencia de lo anterior una buena formación del fruto y la productividad final por metro cuadrado cultivado. Sumado a la superación de malas prácticas sobre el monitoreo y la omisión de estrategias de control de variables, lo cual representan la aparición de plagas, malezas y enfermedades, según la FAO [19] cada año, hasta un 40 por ciento de los cultivos alimentarios en el mundo se pierden a causa de plagas y enfermedades de las plantas, provocando pérdidas anuales en los comercios agrícolas y dando un golpe contundente a los países que dependen en gran porcentaje de la producción agraria y alimentaria.

Siguiendo las tendencias actuales de la industrial 4.0 y de los procesos Agtech, en la planeación de este proyecto se decidió no utilizar una agricultura urbana tradicional, sino una agricultura urbana de precisión basadas en arquitecturas IoT (Internet of things). Procesos considerados ya que en la actualidad adquirir sistemas de agricultura de precisión es demasiado costoso, y más cuando se habla de sistemas satelitales, sensores espectrales e imágenes de suelo infrarrojas. Por lo tanto, si consideramos que un pequeño y mediano productor urbano no tiene la capacidad de adquisición de tecnología y de ayuda técnica constante referente a los últimos estudios, tenemos como política de desarrollo el haber implementado tecnología e ingeniería a bajo costo y para un mundo mejor.

En el mismo orden, demostrar el uso de tecnologías mediadoras como el internet de las cosas (IoT) se plantea como una fuerza para mitigar los efectos del cambio climático en los huertos urbanos y poder generar trazabilidad de datos dependiendo de las condiciones o parámetros ambientales del huerto urbano [20]. De forma tal que en la actualidad más que cultivar un producto y cosechar, es de vital importancia cosechar datos. Ya que estos serán recursos claves para la solución de problemas a futuro. 


\section{MÉTODO}

El diseño y desarrollo del modelo de huertos comunitarios mediados por tecnologías, fue estructurado considerando necesidades identificadas y una visión de co-creación, en la cual la interacción de la comunidad con los investigadores de la IES, permitió avanzar en el desarrollo de tres fases que garantiza la rigurosidad y viabilidad del modelo: primera fase, se establece una investigación aplicada y de campo, lo cual determinó la disponibilidad de capacidades y recursos de la institución del suroccidente de Colombia, así como los criterios de selección de la zona urbana. Una comunidad del distrito de Aguablanca en Santiago de Cali-Colombia, con 16.353 habitantes. Sector en el cual, interactúa la Fundación Autónoma (Fundautónoma) asociada con la IES que promueve el proyecto. Mediante la referida fundación se fomenta el apoyo hacia las comunidades bajo los principios que rigen la sostenibilidad. Como estrategia de mediación, se promueve un modelo integral que fortalezcan las capacidades tanto humanas como sociales para el mejoramiento de la calidad de vida de los habitantes del sector. Este actor, ha permitido la articulación comunidad-academia como estrategia para la viabilidad del proyecto. Con el fin de impulsar el proyecto de forma integral, considerando el fortalecimiento de las redes locales y en coherencia con las dinámicas del territorio, la propuesta de trabajo se viabiliza con diferentes actores que interactúan con la Fundación. La caracterización de los actores se resaltan: primeros actores, dos líderes territoriales ex-pandilleros que desarrollaron huertos, su identificación e interés de vincularlo al proceso es el resultados de este actividades previas asociadas con procesos de resocialización y resignificación de las fronteras invisibles que marcaban los territorios, el segundo actor, fue el grupo de estudiantes de colegios (educación básica y media) vinculados a la fundación. La representación estuvo en siete jóvenes, hombres y mujeres, entre 8 y 19 años en etapa escolar quienes venían desarrollando procesos de acercamiento con el concepto de la siembra en el proyecto "Huerto Escuela" de Fundautonoma y el cual tiene como característica ofrecer a los jóvenes de la comuna, la posibilidad de contar con espacios y dinámicas que los aislen, durante los horarios en los cuales no asisten a clase, de las problemáticas sociales y las redes de delincuencia que allí existen. De igual forma, se resalta la participación en el equipo de una mujer de 49 años, quien se encuentra finalizando el bachillerato. A partir de lo expuesto, se evidencia que los criterios del grupo poblacional, se determinó considerando la heterogeneidad en edades, formación académica (bachillerato), así como la inclusión de mujeres, con saberes previos en los que el contacto con la siembra haya tenido una connotación social y no solo de consecución de alimentos. En el mismo orden, la caracterización de la población se resalta considerando las condiciones de vulnerabilidad económica, social, como también las complejas condiciones ambientales y de disponibilidad de recursos en general para llevar a efecto la siembra y producción de tomate, cilantro, frijol y lechuga, como también diversidad de plantas medicinales en huertos verticales comunitarios.

Como parte de las estrategias emprendidas para promover el fortalecimiento de la transferencia del conocimiento fueron programados talleres formativos sobre el proceso, cuya ejecución se desarrolló por un período de un mes. Actividad que permitió tanto la transferencia de conocimiento, como la detección de necesidades, permitiendo de igual forma el reconocimiento de las capacidades y disponibilidad por parte de la comunidad para emprender y consolidar el proyecto.

Segunda fase, mediante interacciones grupales colaborativas, procesos de comunicación asertivos y el desarrollo asertivo de los talleres programados, se inicia el proceso de transferencia de conocimiento por parte de los investigadores de la IES, quienes fomentan la apropiación de lo conocimiento, utilizando prácticas para la compre nsión sobre las variables del proceso de cultivo y reconocimiento conceptual de las tecnologías. La programación del desarrollo de esta segunda fase, se planificó durante un mes, permitiendo evidenciar la efectiva transferencia- explotación-apropiación del conocimiento, así como las formas de su aplicación y se viabiliza desde una visión de corresponsabilidad que permitió el avance y la viabilidad de la innovación social.

Tercera fase, permitió de forma colaborativa entre universidad-comunidad la aplicación de procesos para el desarrollo, efectividad y futura proyección del modelo de huertos comunitarios, utilizando como base diversas herramientas metodológicas, entre las más destacadas para las fases de diseño, proceso administrativo planeación y ejecución, la cual se complementa del libro de diseño del MITInternational Development Design Summit (IDDS) [21]. Con respecto al monitoreo y la trazabilidad del huerto comunitario, se adoptó el modelo tecnológico de diseño para sistemas IoT (Internet of things), denominada IoT thinking [22]. En el mismo orden, se dio continuidad con el monitoreo y evaluación, previo al registro de los datos adquiridos por medio del sistema de sensores y un datalogger, donde se propusieron dos arquitecturas tecnológicas para determinar la trazabilidad del comportamiento del cultivo y la correlación basada en la técnica de regresión múltiple lineal [23], permitiendo el estudio de las variables agroclimáticas sobre la productividad y operatividad del modelo. Esta fué la fase más extensa, con una duración de dos meses y medio ya que la construcción del elemento fue la actividad que tomó más tiempo. De esta forma se prevé garantizar la efectividad de una innovación social fundamentada en el enfoque de optimización de los recursos requeridos considerando desde el "Input hasta el Output" como estrategia que garantiza la efectividad del huerto mediado por tecnologías y se proyecte en la preservación y sostenibilidad ambiental.

$1^{\text {sh }}$ LACCEI International Multiconference on Entrepreneurship, Innovation and Regional Development - LEIRD 2021: "Ideas to Overcome and Emerge from the Pandemic Crisis", Virtual Edition, December 9 - 10, 2021. 


\section{RESULTADOS DISCUSIÓN Y PROPUESTA DE MODELO}

Como parte de los resultados en una primera fase de desarrollo de la propuesta de solución, se responde a las diferentes fases planeadas para su desarrollo. De esta forma, se obtuvo la identificación de todos los actores de la comunidad, prevaleciendo una diversidad demografía heterogénea en el que participan jóvenes estudiantes de colegios entre 14 y 17 años, líderes de los huertos de la zona entre 25 y 37 años, así como una adulta mayor de 49 años. La diversidad de los perfiles en cuanto a edad, formación y experiencia permitió identificar los intereses tanto de formación como de operatividad de procesos, generando potencialidades para la consolidación de la innovación social. Asimismo, como parte de la experiencia, se logró socializar aprendizajes y experiencia a partir de la diversidad cultural y conocimientos ancestrales de la población, ya que en su mayoría está conformada por personas desplazadas del pacífico colombiano por el conflicto armado o por búsqueda de mejores oportunidades. Es importante resaltar que de manera colectiva se manifestó como oportunidad de innovación social, el deseo por superar la estigmatización de la cual son víctimas los habitantes de la comuna 13 por el resto de la ciudad. Entre las estrategias consideradas para la transferencia del conocimiento tecnológico, se contempló el aprovechamiento de las huertas ya existentes que ameritan ser renovadas a partir del uso de tecnologías que garanticen procesos productivos sostenibles que conlleven a contribuir en la seguridad alimentaria

Como resultados de la segunda fase, se tiene como consigna que en el último año un alto porcentaje de proyectos agrícolas se diseñan con base a investigaciones previas o referentes de orden mundial, que adoptan las tecnologías en los huertos urbanos. Sin considerar que la tasa de adopción de estas tecnologías en el mayor de los casos es muy baja debido a que el producto desarrollado no siempre corresponde a las necesidades del productor ni a su capacidad adquisitiva, para hacer efectiva la solución a sus problemas. Sumado a que la variabilidad de los suelos no es la misma en todas las demografías, es por eso, de vital importancia conocer e identificar el contexto de cómo influyen las variables agroclimáticas de cualquier cultivo, sus plagas y enfermedades más comunes. A partir de lo expuesto, como plan de acción se desarrolla una serie de capacitaciones e interacciones grupales que permiten transferir conocimiento georeferenciado (In-situ) entendiendo muy bien el comportamiento de las variables de los procesos agrícolas y así mitigar los efectos del cambio climático de una forma efectiva.

En el mismo orden, se hace referencia sobre la importancia de la transferencia del conocimiento a partir de metodologías participativas como mecanismo que permite superar las brechas existentes entre el huertero y la tecnología, sin dicha participación la apropiación social de los conocimientos sería nula. Al conceptualizar mediante grupos de trabajo, temas sobre agricultura y de cómo influyen variables en los cultivos, los participantes conocieron términos tales como ¿Qué es una variable?, ¿Qué son las variables físicas?, ¿Qué son las señales análogas y digitales?, ¿Qué es un microprocesador?, entre otros conceptos que son de vital importancia para comprender el despliegue tecnológico.

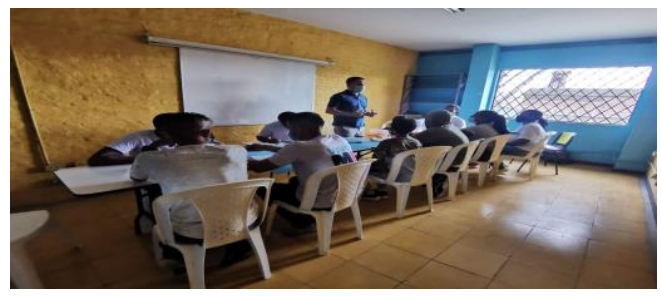

Fig. 1 Capacitaciones de transferencia del conocimiento tecnológico

Se realizaron actividades teórico-prácticas, tales como fundamentos de programación, adquisición de señales, se hicieron ejemplos reales con frutas cítricas, ácidas, tierra seca, tierra humedad, para aprender a calibrar los instrumentos de medición o sensores, tema importante para cumplir con el requerimiento de confiabilidad y precisión de los datos. Al finalizar las diferentes capacitaciones, se realizaron prácticas en grupos para lograr los aprendizajes y el análisis de datos obtenidos a través de los sensores permitiendo su escritura manual para la toma de decisiones dependiendo del tipo de cultivo. Al respecto, los resultados son positivos al observar como el dominio del lenguaje en los huerteros se transforma y se evidencia el empoderamiento de ellos hacia la tecnología como mediadora para optimizar los cultivos. Finalmente se explica cómo será la siguiente fase de desarrollo y todo el despliegue logístico-técnico

Con respecto a los resultados de la tercera fase, la aplicación de una metodología mixta, permitió obtener información de tipo social, co-diseño, co-producción, desarrollo tecnológico y apropiación del conocimiento de manera técnica y participativa. Asimismo, mediante las jornadas de reconocimiento de necesidades se definió el mejorara el contexto del poblado y la percepción de este ante el resto de la ciudad. En el mismo orden, el proceso de ideación se consolidó mediante sesiones de generación de valor, en la que prevalecieron procesos creativos para el desarrollo del modelo, mediante herramientas de: a) bisociación [24], combinando un contenedor con una carreta de reciclaje, b) cambio de perspectiva, dándole otro enfoque a el uso del contenedor plastico, c) ideación a partir de un objeto, en esta actividad se tomó la cama del huerto, re-enfocándose lo cual permitió diversidad de usos al combinarlo con otros objetos que permiten su transformación. De esta forma, se prevé el uso de la cama de huerto para actividades diferentes a su uso convencional, por último, se implementó la generación de ideas utilizando la técnica de SCAMPER [25]. De estas tres actividades de ideación, se generaron setenta y una (71) ideas a trabajar, de las cuales se seleccionaron como punto de partida veinte (20). Posterior a estas, se desarrollaron ocho (8) bocetos, ver Fig. 2. 

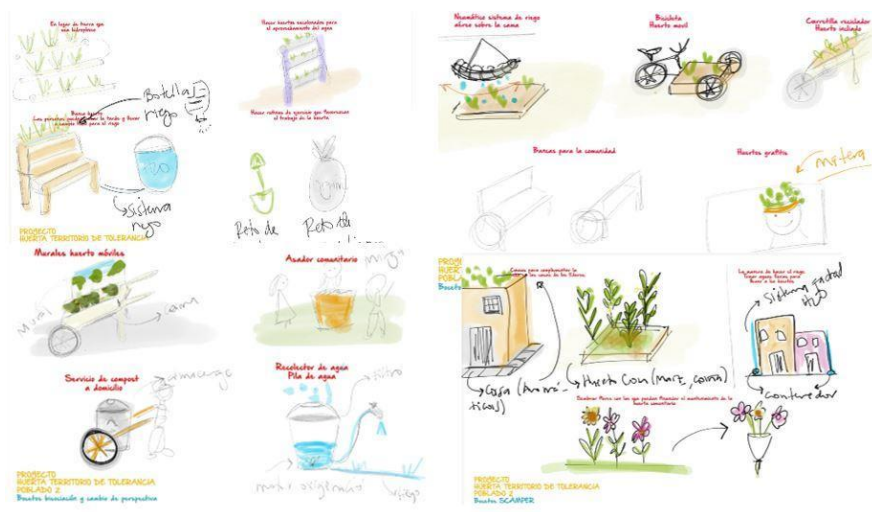

Fig. 2. Bocetos sobre propuestas de diseño

Una vez presentados los bocetos a la comunidad, para la selección de la alternativa de solución se implementó una matriz de ponderación para valorar los pros y contras de cada propuesta, ver Tabla 1.

TABLA I

Pros y Contras

Selección Alternativas De Diseño

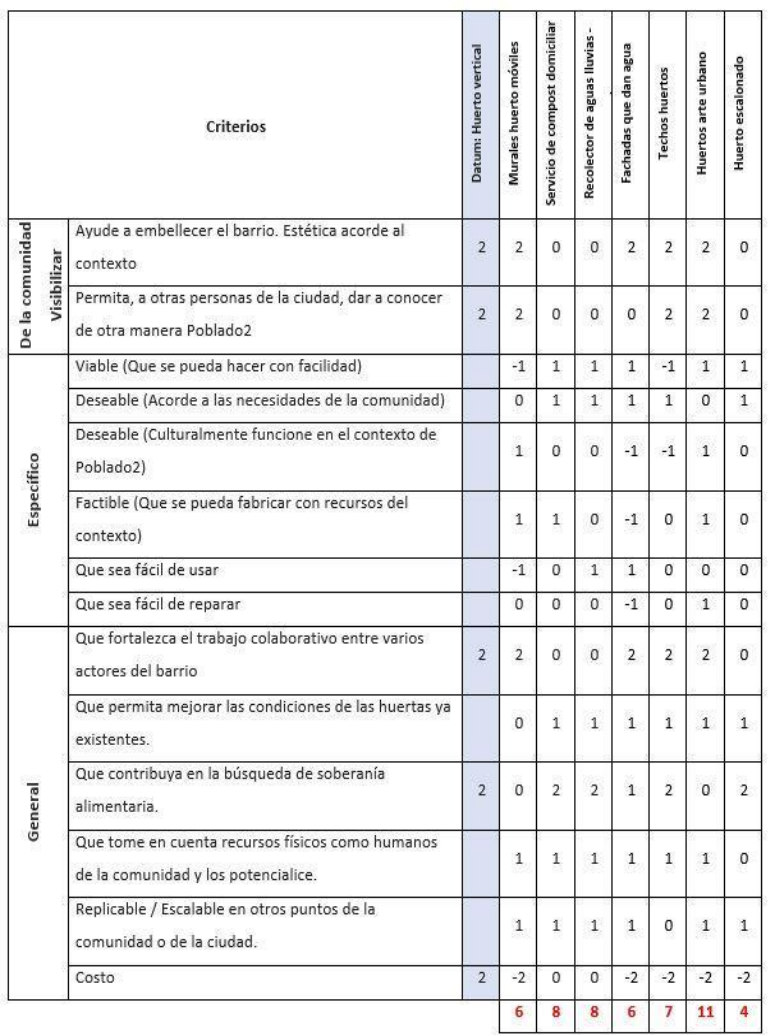

A partir de catorce (14) criterios se seleccionó como alternativa de solución el desarrollo de un huerto vertical con sistema de riego autónomo, para la producción de plantas que atraigan el tránsito de animales polinizadores, que a su vez permita la cosecha de hierbas como el cilantro y plantas medicinales, hortalizas de hojas como la lechuga, acelga y rúcula, granos como el frijol y frutos como el tomate. Para el perfilamiento de esta propuesta, se realizó una sesión preliminar, en la cual se definieron los requerimientos de diseño acorde al espacio de la fundación donde se iba a realizar el prototipo de validación, el tipo de plantas a cultivar y las condiciones climáticas. Como requerimientos propios del diseño y su funcionalidad, se definió que debía permitir un adecuado riego y por ende la instalación de la manguera de riego de $0.5 \mathrm{~cm}$, flujo del agua en la zona de la siembra, de igual forma la identificación de puntos hídricos y de energía, microprocesadores de bajo costo, sensores confiables y con soporte a la intemperie. Para la siembra de las hortalizas debía haber una superficie de soporte con profundidad de $40 \mathrm{cms}$, acceso al sol y la posibilidad de garantizar su crecimiento vertical. En cuanto a las plantas como el cilantro, medicinales y flores, se definió que debían garantizar una superficie de soporte y con profundidad de $8 \mathrm{~cm}$. de diámetro. Una vez se definieron estas características, se identificaron los materiales disponibles en el contexto, entre estos tubos de pvc, "zuncho plástico" y aerosoles, cuya disponibilidad se obtuvo a través de la IES; tarros de pintura, botellas de gaseosa y baldes que estaban desechados en la fundación. Con los requerimientos descritos, cada integrante inició su proceso de bocetación, eligiendo como propuesta seleccionada, acorde al cumplimiento de los requerimientos ya planteados, el desarrollo de la figura. 3.

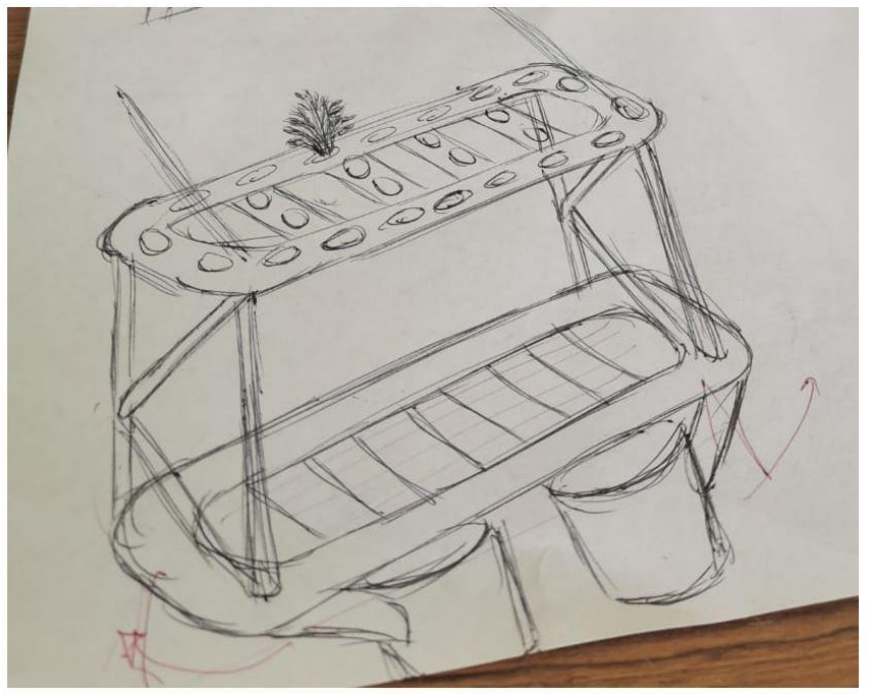

Fig. 3. Propuesta de diseño seleccionada para la fase de prototipado.

Una vez definido el diseño, se realizaron ajustes técnicos considerando medidas, uniones y ensambles acorde a la tecnología disponible para la construcción del prototipo, ver Figura 4.

$1^{\text {sh }}$ LACCEI International Multiconference on Entrepreneurship, Innovation and Regional Development - LEIRD 2021: "Ideas to Overcome and Emerge from the Pandemic Crisis", Virtual Edition, December 9 - 10, 2021. 

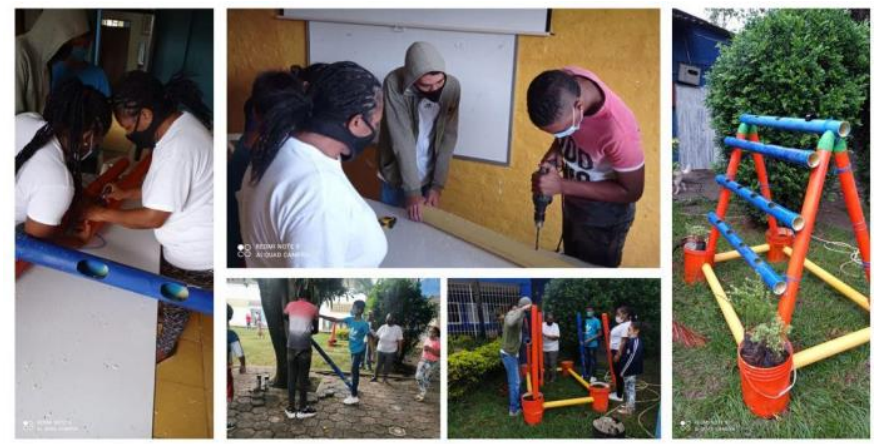

Fig. 4. Desarrollo de prototipo.

Considerando el pensamiento de internet de las cosas o IoT thinking, fue relevante tomar en cuenta aspectos culturales para que el lenguaje de la tecnología fuera intuitivo para el perfil de la comunidad con la que se interactúa, permitiendo de esta forma la apropiación del diseño del sistema tecnológico. A través de la aplicación de la metodología IoT thinking se analizaron los procesos de conectividad, las condiciones del entorno, el testeo y validación del sistema, con el fin de proponer y desarrollar arquitecturas de monitoreo y control de manera local y remota que le apunten a la apropiación y accesibilidad de tecnología de bajo costo para los huerteros del contexto de estudio. A continuación las propuestas de arquitecturas para la implementación de tecnologías mediadoras con IoT en huertos urbanos. Recordemos que, la implementación de tecnología no es para reemplazar al productor o huertero, es un apoyo más dentro de la agricultura tradicional que se ha estado implementando.

\section{A. Arquitectura para el monitoreo de huertos de manera local}

Para el desarrollo de la arquitectura de monitoreo local, LAN (Local Area Network), se tuvo en cuenta requerimientos tales como 1) accesibilidad a las redes (Internet), 2) factibilidad de dispositivo inteligente o smartphone con bluetooth 3) fácil uso de tecnologías 4) infraestructura tecnológicas accesibles en la fundación 5) confiabilidad de los datos 6) dimensiones del huerto 7) optimización y consumo de energías y 8) registro de datos en tiempo real. El sistema funciona a través de un microcontrolador RP2040, raspberry pi pico, donde mediante protocolos $\mathrm{I} 2 \mathrm{C}$ y conversores de análogo a digital se toman los datos provenientes de los sensores, finalmente los datos son visualizados mediante una pantalla LCD y se envían los mediante serial y bluetooth a un aplicativo móvil y de escritorio hecho en software labview. Ver Figura 5.

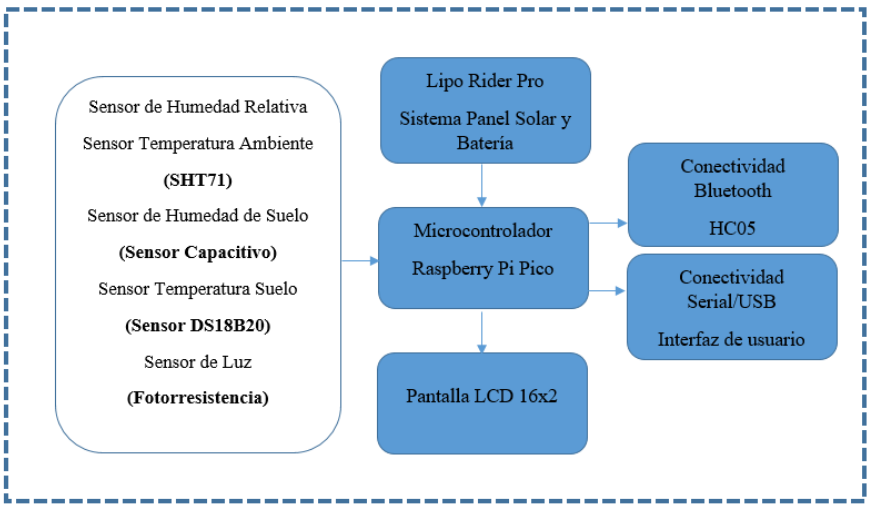

Fig. 5 Arquitectura hardware local

Con el fin de cumplir con uno de los requerimientos importantes como lo es el bajo consumo, el sistema consta de un módulo con panel solar y batería de litio, para poder ahorrar energía e impactar positivamente en el ambiente. Finalmente en la fig. 6 , se visualizan los datos en tiempo real a través de una interfaz intuitiva y visual del huerto urbano.

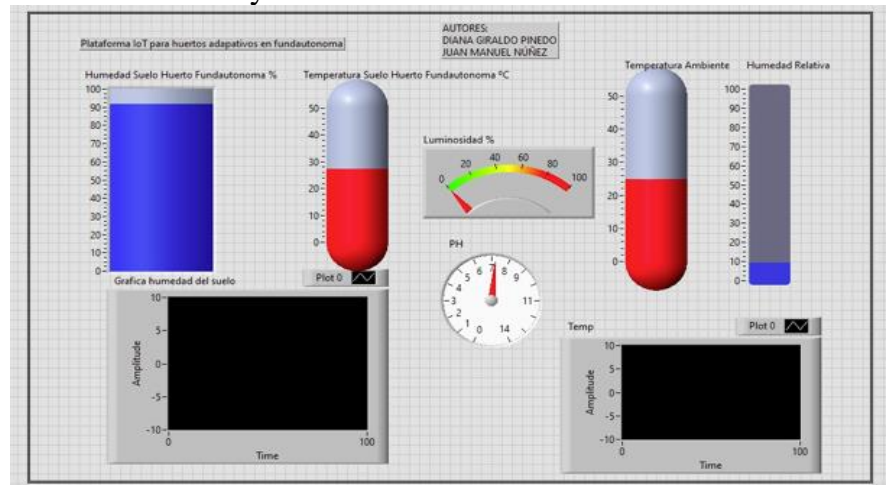

Fig. 6 Intefaz gráfica local para la visualización de datos

\section{B. Arquitectura para el monitoreo de huertos de manera remota}

La conectividad juega un papel muy importante en el uso de arquitecturas de IoT para la agricultura, poder aportar al AgTech y a la industria 4.0. La arquitectura propuesta para este huerto urbano consta de un módulo microcontrolado llamado Node MCU ESP8266, el cual tiene flexibilidad en su programación e incorpora una conexión a la nube mediante el protocolo WiFi, ver Figura 7. 


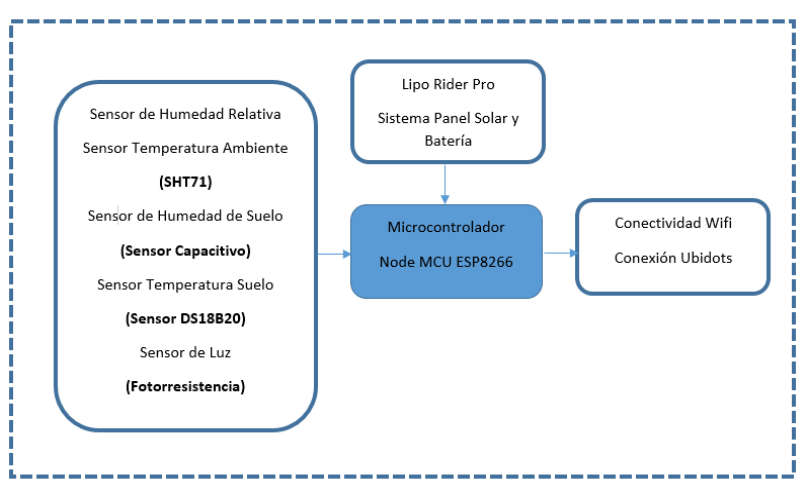

Fig. 7 Arquitectura IoT Propuesta

Es muy importante el uso de arquitecturas de IoT (internet de las cosas), por considerar que la omisión de procesos de medición impide la efectividad de controles,por ende el hecho que se realicen mediciones precisas y los huerteros no tomen acción, es como si se siguiera en la agricultura tradicional. La conexión con la nube se hizo mediante la plataforma Ubidots, en donde por su dinamismo y flexibilidad para el usuario, se pueden crear widgets muy interactivos y poder crear una buena relación hombre-máquina. Gracias al protocolo MQTT y el uso de sockets web, se pueden subir de buena forma y rápida los datos a la nube como se aprecia en la Fig. 8. Ver arquitectura física de los elementos Fig. 9.

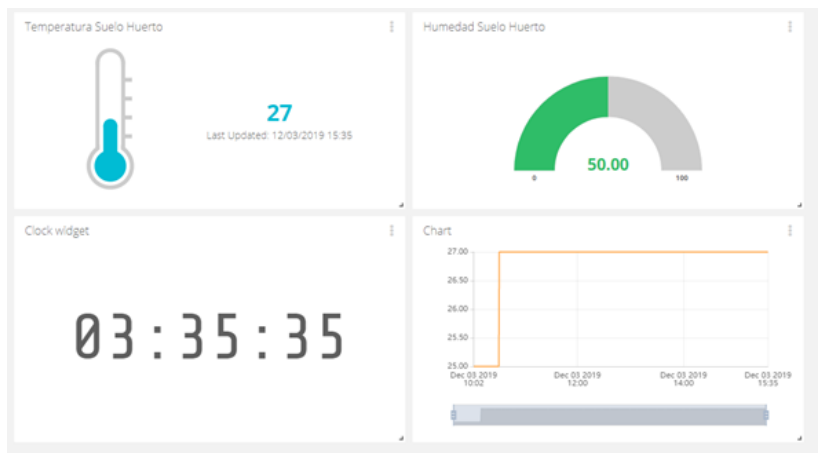

Fig. 8 Plataforma en la nube (Ubidots)

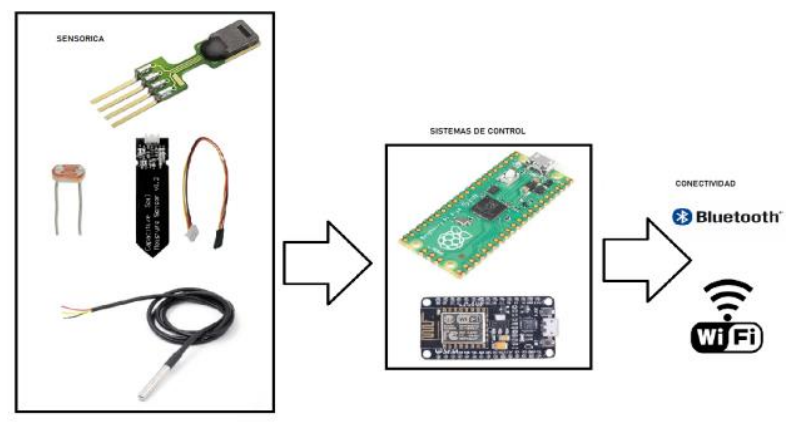

Fig. 9 Arquitectura elementos físicos IoT
Como resultado del proceso participativo con la comunidad y todo el proceso de co-diseño, se generó una apropiación de la interfaz del usuario, generando requerimientos por la misma comunidad para poder entender e interpretar cada uno de los mandos y datos presentados por la dashboard. Todo lo anterior, se condensa en talleres participativos continuos, prácticas de laboratorio en casa y OpenLabs con los usuarios participantes del proyecto. Al respecto, se resalta que la metodología de diseño fue concebida para ser replicada considerando todas las fases del proceso. De esta forma, se garantiza un diseño con características accesibles, factible y medible como estrategia que contribuye a que las comunidades no solo, se apropien de del proceso, sino que se conviertan en multiplicadores del proceso hacia otras comunidades, resaltando de esta forma el potencial innovador de las regiones en materia de huertos urbanos.

\section{Desarrollo del sistema de riego}

Se realizó un sistema de riego, ver Fig. 8, basado en goteo y aspersión, donde la comunidad realizó todo el sistema completo de recirculación de agua e instalación. El sistema de riego se puede activar de manera manual con la arquitectura local e IoT, y se puede generar horarios de riego de manera automática con base al estado actual de los sensores de humedad y temperatura.

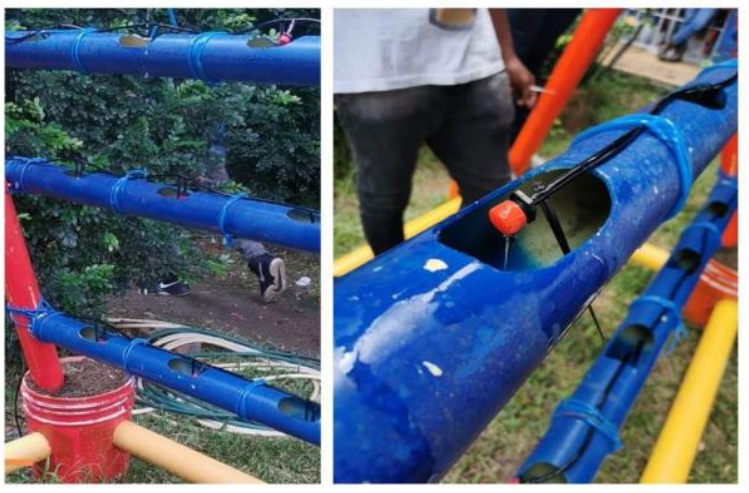

Fig. 10 Sistema de riego

De esta manera se evidencia como en el desarrollo del prototipo, desde la ideación hasta la apropiación de la tecnología la comunidad tuvo acceso a conocimientos propios de la ingeniería y del diseño de objetos de manera práctica, validando el aprender haciendo como una estrategia para romper las brechas de acceso a este tipo de formación para las comunidades vulnerables.

Como parte estratégica del proyecto que garantiza su factibilidad, fueron definidos los indicadores cuantitativos que permiten monitorear y definir los indicadores de control, desde la medición de capacidades en cuanto a la apropiación de los conocimientos tecnológicos, como también la respuestas del equipo consolidado para avanzar en la construcción, tanto del huerto como del sistema que este requiere. Es así, como lograr

$1^{\text {sh }}$ LACCEI International Multiconference on Entrepreneurship, Innovation and Regional Development - LEIRD 2021: "Ideas to Overcome and Emerge from the Pandemic Crisis", Virtual Edition, December 9 - 10, 2021. 
presentar resultados tangibles que modela la práctica relacionada con los aprendizajes según lo programado en las fases previas. De igual forma, los indicadores responden a los criterios de base para el diseño de un huerto. Sumado, a la identificación de los recursos necesarios para la construcción del huerto entre estas: herramientas básicas como taladros y caladoras, la instalación del sistema de riego para el elemento, de sensores de humedad relativa, temperatura ambiente y del suelo, temperatura del suelo, luminosidad y lluvia, instalación de panel solar para el abastecimiento de energía del sistema y el desarrollo del display con el que el grupo podrá recolectar los datos. Por otra parte, los criterios básicos medioambientales como la optimización del uso del recurso hídrico, al igual que la reducción de pérdida de plantas que se dan actualmente por inadecuadas condiciones térmicas y de cuidado es otra de las mediciones de relevancia.

Como indicadores cualitativos, en un mediano o largo plazo, se resalta la transformación de los perfiles de los actores sociales participantes lo cual se verá reflejado en nuevas oportunidades formativas y de mejores ofertas de empleabilidad a partir de los saberes y experiencias apropiadas. Otras de las mediciones se representan en el fortalecimiento de los roles de líderes comunitarios, la posibilidad de mitigar las condiciones de hambre de la comunidad, el fortalecimiento de redes entre los actores locales y la reducción de la incidencia de los jóvenes y líderes en grupos delincuenciales.

En líneas generales, los avances alcanzados permiten ser referentes para nuevos desarrollos, emprendimientos y su sostenibilidad. En el largo plazo se prevé, poder medir la capacidad productiva del huerto y su aporte en la reducción del hambre.

\section{CONCLUSIONES}

Determinar como la transferencia de conocimiento e innovación social en el desarrollo de huertos comunitarios mediados por tecnologías, logró consolidarse como parte de una dinámica de ejercicio práctico y de co-creación, permitió concluir sobre la importancia de las interacciones desde las IES mediante procesos efectivos de transferencia de conocimiento para con las comunidades. Al respecto se evidenció, cómo se incentivan los miembros de las comunidades desde el momento de ser parte de proyectos y programas en los cuales, tanto el conocimiento como la innovación social, se articulan para consolidar propuestas que redundan tanto en el mejoramiento continuo de procesos centrados en la calidad de vida, como en convertirse en referentes para ser adaptados en diversas comunidades que se transforman a partir de sus propias experiencias.

Entre los aspectos relevantes del estudio, se destaca la efectiva transferencia de conocimiento mediante estrategias centradas en el aprender haciendo y el aprendizaje a lo largo de la vida [26]. Sumado a la capacidad de apropiarse de conceptos técnicos y de ingeniería en una población de 16.353 habitantes, que solo cuenta con una institución educativa para 3.900 jóvenes entre los 5 y los 19 años, lo cual representa solo el 23,9\% de la población con asistencia escolar [27].

Sobre lo expuesto, la importancia del estudio se fundamenta en el alcance del diseño para la innovación social desde la visión de inclusión que permitió procesos de interacción con diferentes miembros de las comunidades para consolidar el enfoque de co-creación y cubrir necesidades a partir de prácticas de proyectos que garantizan la factibilidad y viabilidad. De igual forma, la corresponsabilidad entre las IESSociedad, vista desde la la capacidad de transferencia de conocimiento e innovación social, prevé fortalecer estructuras de proyectos y modelos como el de huertos mediado con IoT, a través de los cuales la participación de jóvenes, se plantea como una forma de incluirlos a un sistema productivo agrícola urbano sostenible, por considerar que el diseño y adaptación de tecnologías de bajo costo, hacen que en los últimos cinco años sea posible pasar del servicio a la creación de productos tecnológicos hechos en Colombia, lo cual permitirá que con cada desarrollo se reduzcan las brechas digitales existentes entre huerteros tradicionales y la tecnología.

De igual forma, se hace referencia sobre emprender estrategias para mitigar el cambio climático, considerando la adopción de tecnologías de la información y comunicación, como recursos que permiten la trazabilidad del proceso productivo agrícola y por ende minimizar el impacto de pérdidas mediante actuaciones oportunas que permiten cambios cualitativos y cuantitativos sobre la producción y cosecha de hierbas como el cilantro y plantas medicinales, hortalizas de hojas como la lechuga, acelga y rúcula, granos como el frijol y frutos como el tomate. Desde la perspectiva de lo expuesto, se fomentan acciones para prever el relevo generacional en las familias de productores y por ende minimizar las brechas que generan la crisis alimentaria proyectada para el 2050 [18].

Entre las implicaciones prácticas del modelo de huertos mediados por tecnologías, se resalta la capacidad de interacción de los miembros de la comunidad y las formas de apropiarse del conocimiento para generar valor y dar continuidad a futuros proyectos que desde la innovación social se consoliden en emprendimientos con características sostenibles. Asimismo las estructuras consolidadas a partir del conocimiento y la práctica fueron valoradas para mediar entre las comunidades huerteras vulnerables, que consideran entre sus prioridades los efectos del cambio climático, sin embargo desconocen las tendencias tecnológicas que conllevan a nuevas formas de hacer agricultura inteligente.

Como líneas futuras asociadas al estudio, se resalta el fortalecimiento simultáneo entre la utilidad de los sistemas y tecnologías como estrategia que demanda la trazabilidad de procesos productivos en huertos comunitarios, así como generar líneas asociadas con la democratización de las

$1^{\text {sh }}$ LACCEI International Multiconference on Entrepreneurship, Innovation and Regional Development - LEIRD 2021: "Ideas to Overcome and Emerge from the Pandemic Crisis", Virtual Edition, December 9-10, 2021. 
tecnologías para el desarrollo de procesos agrícolas, lo cual prevé su vinculación con la sistematización de la gestión de investigación, innovación y emprendimiento en la que interactúan las denominadas 5 hélices, lo cual contribuye a minimizar riesgos y complejidades inherentes a la apropiación y puesta en práctica de la producción de conocimiento científico-tecnológico.

\section{REFERENCIAS}

[1] R. Arocena and J. Sutz, "Universities and social innovation for global sustainable development as seen from the south," Technological Forecasting and Social Change, vol. 162, p. 120399, Jan. 2021.

[2] S. Banerjee, L. Lucas dos Santos, and L. Hulgård, ">Intersectional knowledge as rural social innovation," Journal of Rural Studies, May 2021.

[3] I. Nonaka, "A Dynamic Theory of Organizational Knowledge Creation," Organization Science, vol. 5, no. 1, pp. 14-37, Feb. 1994.

[4] J.-C. Spender, "Making knowledge the basis of a dynamic theory of the firm," Strategic Management Journal, vol. 17, no. S2, pp. 45-62, Dec. 1996.

[5] T. H. Davenport and L. Prusak, "Working knowledge," Ubiquity, vol. 2000, no. August, p. 2, Aug. 2000.

[6] C. Liyanage, T. Elhag, T. Ballal, and Q. Li, "Knowledge communication and translation - a knowledge transfer model," Journal of Knowledge Management, vol. 13, no. 3, pp. 118-131, May 2009.

[7] Nonaka, I., y Takeuchi, H. "The Knowledge Creating Company". Oxford University Press, Oxford.(1995).

[8] G. Mildenberger, G. Schimpf, and J. Streicher, "Social Innovation Assessment? Reflections on the impacts of social innovation on society Outcomes of a systematic literature review," European Public \& Social Innovation Review, vol. 5, no. 2, pp. 1-13, Dec. 2020.

[9] O. Reshetnyak and R. Lobodin, "CLUSTERS IN INNOVATION: TRIPLE HELIX, QUADRUPLE HELIX AND QUINTUPLE HELIX MODELS," Odessa National University Herald. Economy, vol. 25, no. 6(85), 2020.

[10] H. Paredes-Frigolett, "Modeling the effect of responsible research and innovation in quadruple helix innovation systems," Technological Forecasting and Social Change, vol. 110, pp. 126-133, Sep. 2016.

[11] E. G. Carayannis and D. F. J. Campbell, "Les systèmes d'innovation de la quadruple et de la quintuple hélice," Innovations, vol. 54, no. 3, p. 173, 2017.

[12] Organización de Naciones Unidas. "Informe de la Comisión Mundial sobre el Medio Ambiente y el Desarrollo" Asamble General. 1987-

[13] A. Papuziński, "Business ethics of Brundtland report," Scientific Papers of Silesian University of Technology. Organization and Management Series, vol. 2017, no. 104, pp. 329-338, 2017.

[14] Margolin, V. "Construir un mundo mejor - Diseño y responsabilidad social”. Designio. México. (2017).

[15] Papanek, V. "Diseño para el mundo real. Ecología humana y cambio social". Academy Chicago Publishers. Chicago. (1971).

[16] Manzini E. "Cuando todos diseñan, una introducción al diseño para la innovación social". Fedrigoni. Madrid. 2015.

[17] Ministerio de Agricultura de Colombia. "Censo Agropecuario". Gobierno de Colombia. 2015.

[18] Dirección de Administración Nacional de Estadística. "III censo Nacional Agropecuario de Colombia". 2014.

[19] Organización de Naciones Unidas para la Alimentación y la Agricultura FAO. Año Internacional de la Sanidad Vegetal. 2020.

[20] A. K. Podder, A. A. Bukhari, S. Islam, S. Mia, M. A. Mohammed, N. M. Kumar, K. Cengiz, and K. H. Abdulkareem, "IoT based smart agrotech system for verification of Urban farming parameters," Microprocessors and Microsystems, vol. 82, p. 104025, Apr. 2021.

[21] Smith A., \& Linder B. Manual Construcción de la Capacidad Creativa. Libro de diseño. MIT D-lab. (2016)

[22] J. M. Núñez V., V. L. Vargas and Y. M. Quezada L., "Implementation of a participatory methodology based on STEAM for the transfer of ICT knowledge and creation of Agtech spaces for the co-design of solutions that contribute to the development of small and medium agricultural producers in Colombia, Panama and China," 2020 IEEE World Conference on Engineering
Education (EDUNINE), 2020, pp. 1-6, doi: 10.1109/EDUNINE48860.2020.9149486.

[23] J. M. Núñez V., F. Fonthal R. and Y. M. Quezada L., "Design and implementation of WSN for precision agriculture in white cabbage crops," 2017 IEEE XXIV International Conference on Electronics, Electrical Engineering and Computing (INTERCON), 2017, pp. 1-4, doi: 10.1109/INTERCON.2017.8079671.

[24] Rodriguez, G, "Bisociaciones creativas, sinéctica y pensamiento divergente" Revista Q, Vol. 8. N15. 2013.

[25] O. Serrat, "The SCAMPER Technique," Knowledge Solutions, pp. 311$314,2017$.

[26] Trejos Zelaya, "Aprender haciendo, colaborativamente". Revista SISTEMAS, no. 158, pp. 8-17, 2021.

[26] Alcaldía de Santiago de Cali. Secretaria de Bienestar Social. Ficha de caracterización económica de los barrios de Santiago de Cali. Diagnóstico descriptivo. Poblado 2. 2015.

$1^{\text {sh }}$ LACCEI International Multiconference on Entrepreneurship, Innovation and Regional Development - LEIRD 2021: "Ideas to Overcome and Emerge from the Pandemic Crisis", Virtual Edition, December 9 - 10, 2021. 